\title{
Tunable light beam steering device using polymer stabilized blue phase liquid crystals
}

\author{
Pankaj Joshi, ${ }^{* 1}$ Oliver Willekens ${ }^{2}$, Xiaobing Shang ${ }^{1}$, Jelle De Smet ${ }^{1}$, Dieter Cuypers ${ }^{1}$, Geert Vansteenberge ${ }^{1}$, Jeroen \\ Beeckman $^{2}$, Kristiaan Neyts ${ }^{2}$, Herbert De Smet ${ }^{1}$ \\ ${ }^{1}$ Centre for Microsystems Technology, Ghent University and IMEC, Belgium, \\ ${ }^{2}$ Liquid Crystals and Photonics Group, Ghent University, Belgium.
}

Received February 14, 2017; accepted March 08, 2017; published March 31, 2017

\begin{abstract}
A polarization independent and fast electrically switchable beam steering device is presented, based on a surface relief grating combined with polymer stabilized blue phase liquid crystals. Switching on and off times are both less than 2 milliseconds. The prospects of further improvements are discussed.
\end{abstract}

Blue phases (BP) are thermodynamically stable phases present in some highly chiral liquid crystals between chiral nematic $\left(\mathrm{N}^{*}\right)$ and isotropic phases [1]. The temperature range in which $\mathrm{BP}$ appears in nature is limited to a few degrees Celsius only, which has rendered their study cumbersome and their practical application next to impossible. However, the advent of the polymer stabilization technique, which successfully broadens the temperature range of BP to several tens of degrees Celsius while keeping its dynamic properties, has brought them at the centre of attention of researchers both in academia and in industry [2]. Polymer stabilized blue phase liquid crystals (PSBP-LC) have emerged as a possible candidate material for future liquid crystal material display and in the year 2007 Samsung demonstrated a prototype display as well [3].

There are several advantages of using PSBP-LCs over conventional nematic liquid crystals, viz. non-requirement of alignment layers (making fabrication process simpler and consequentially less expensive), high Kerr constant values, at least one order faster switching speeds, possibility of realizing polarization independent devices et cetera [4-11]. Several non-display devices have also been proposed and experimentally demonstrated, viz. microlenses, phase-gratings, tunable lasers, etc. [12-18]. Among non-display applications of liquid crystals (LC), nonmechanical light steering devices are of great interest [1920]. Various gratings have been used for imparting tunable phase retardation to the phase of transmitted light. The idea of tunable gratings has been thoroughly investigated in the context of holographic polymer dispersed liquid crystals as well [21-25]. Such volume gratings are valuable components in the field of free-space

*E-mail: pankaj.joshi@schaeffler.com optical communications, fiber optics, integrated optics, sensor systems, optical data storage, and optical computers [26-27].

In this letter we present large period $(\Lambda / \lambda \approx 80$, where $\Lambda$ is the period, and $\lambda$ is the wavelength of light used to characterize the device) surface relief polymer gratings topped by a layer of PSBP-LC in which the phenomenon of diffraction and refraction are controlled by manipulating the refractive index of the LCs. The schematics of the device are shown in Fig. 1a. The polymer gratings have a period of $50 \mu \mathrm{m}$, a shallow blaze angle measuring $10^{\circ}$ and a blaze height of $8 \mu \mathrm{m}$. These gratings were fabricated by soft embossing of UV curable glue (NOA 74, from Norland Adhesives, USA). The details about fabrication are described elsewhere [28]. The top of the polymer surface and the counter ITO electrode are separated by Teflon spacer balls of $10 \mu \mathrm{m}$ diameter. The layer of PSBP-LC is sandwiched between the top grating surface and the ITO glass.

PSBP-LC is macroscopically isotropic but anisotropy can be induced by application of an electric field due to the third order nonlinearity, the DC Kerr effect. The induced nonlinearity is given by, what is known as the extended Kerr effect, as given by Eq. 1 [29]:

$$
\Delta n(E)=\Delta n_{s}\left(1-\exp \left[-\left(\frac{E}{E_{S}}\right)^{2}\right]\right),
$$

where, $\Delta n_{s}$ and $E_{s}$ are the saturation anisotropy and saturation electric field, respectively, which are obtained by performing a fitting to experimentally obtained data. The liquid crystal mixture consists of nematic LCs HBG980000-00 (from HCCH, China) 84.95 wt.\%, chiral dopant R5011 (from $\mathrm{HCCH}$, China) 3.33 wt.\%, monomer EHA (from Synthon, Germany) 4 wt.\%, cross-linker RM257 (from Merck, Germany) 7.1 wt.\% and photoinitiator DMPAP (form Merck, Germany 0.62 wt.\%). The LC cell was assembled by dispensing a UV curable glue mixed with $10 \mu \mathrm{m}$ spacer balls, circumscribing the grating area but leaving two openings for filling the LC mixture. 
To ensure a uniform cell gap, the cell was placed in a pressure chamber during UV illumination. The mixture was infiltrated in the LC cell by capillary filling at room temperature. The sample was heated to the isotropic state $\left(70^{\circ} \mathrm{C}\right.$ ) and slowly cooled down at a rate of $0.1^{\circ} \mathrm{C} / \mathrm{min}$ to $61.5^{\circ} \mathrm{C}$. Five thermal cycles were applied at a cooling/heating rate of $0.1^{\circ} \mathrm{C} / \mathrm{min}$ to enlarge the $\mathrm{BP}$ domain sizes [30]. The $\mathrm{BP}$ was polymer stabilized at $62^{\circ} \mathrm{C}$, with UV intensity $50 \mathrm{~mW} / \mathrm{cm}^{2}$ for $8 \mathrm{~s}$ and the resulting texture is shown in Fig. $1 \mathrm{~b}$.

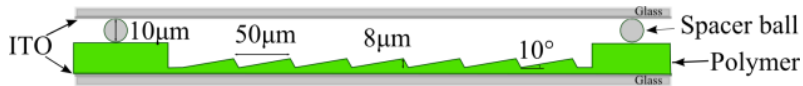

(a)

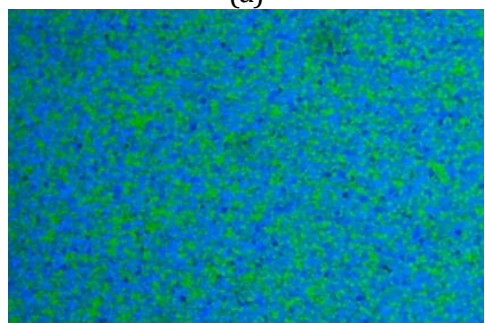

(b)

Fig. 1. (a) The schematics of the tunable surface relief grating device (image is not to scale), (b) Texture of polymer stabilized blue phase liquid crystals on top of a polymer grating observed between crossed polarizers.

The nematic LC HBG980000-00 has $\Delta n=0.141$, dielectric anisotropy $\Delta \varepsilon=+34.3$ and average dielectric anisotropy $\langle\varepsilon\rangle=22.4$. It is worth mentioning that a thin layer $(\sim 100 \mathrm{~nm})$ of $\mathrm{SiO}_{2}$ was deposited on top of the gratings by a PECVD process. It was found that without a barrier layer the polymer stabilization process failed as soon as the sample was exposed to UV. One possible reason for this could be the detrimental interaction between the acrylate molecules of the NOA glue and the acrylates used to polymer stabilize the blue phase. When using much inert $\mathrm{COC}$, we faced no problems with polymer stabilization. The $\mathrm{SiO}_{2}$ barrier layer precludes such interactions, thereby facilitating successful polymer stabilization.
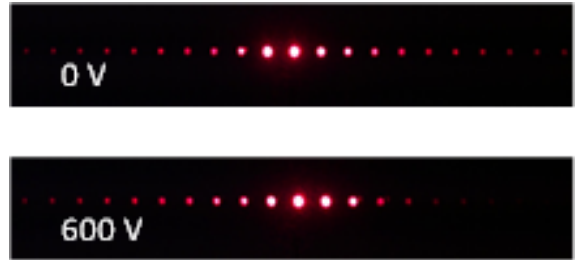

Fig. 2. Diffraction pattern of the grating at $633 \mathrm{~nm}$ wavelength with and without voltage applied.

The gratings were characterized by using a He-Ne Red laser with wavelength $633 \mathrm{~nm}$, as this wavelength lies beyond the Bragg reflection wavelength range of the $\mathrm{BP}$ used in this study. Figure 3 shows the variation in power of the $0^{\text {th }}, 1^{\text {st }}$ and $-1^{\text {st }}$ orders as an AC voltage at $1 \mathrm{kHz}$ was applied. The light was incident upon from the flat side of the gratings and the power of the individual orders was measured (by blocking other orders with an iris) with an avalanche photo-diode (C 5460-01, from Hamamatsu, Japan). The linearly polarized laser was followed by a half wave plate to control the polarization state of the light passing through the gratings. As PSBP is macroscopically isotropic, its index ellipsoid is spherical in shape, and when the voltage is applied it elongates along the field lines owing to the positive dielectric anisotropy of the base nematic liquid crystals in the BP. As a first order approximation it can be assumed that the field lines in the LC region are oriented perpendicularly to the substrates which is also the direction of light propagation, and hence the refractive index experienced by the light is basically $n_{o}$, which decreases monotonically as a function of the applied field, as can be seen from Eq. 2. [31]:

$$
n_{o}(E)=n_{i s o}-\frac{\Delta n}{3}
$$

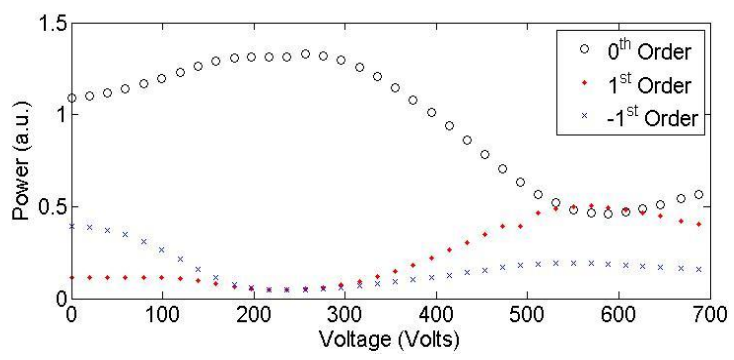

Fig. 3. Variation of power of $0^{\text {th }}, 1^{\text {st }}$ and $-1^{\text {st }}$ diffraction order as a function of voltage applied to the grating.

As can be seen from Fig. 3, the power in the $0^{\text {th }}$ order first increases as the voltage is increased to $250 \mathrm{~V}$. This is because the average refractive index of the PSBP-LC is slightly larger than that of the glue used to fabricate the gratings, and around $250 \mathrm{~V}$ the refractive indices match, causing most of the light to go through with minimal diffraction. The decrease in the refractive index contrast is apparent as the number of diffraction orders visible to the naked eyes goes down. As the voltage is further increased, the refractive index decreases further and the power in the $0^{\text {th }}$ order goes down. At $580 \mathrm{~V}$ the power in the $0^{\text {th }}$ order reaches its minimum while that in the 1 st order reaches its maximum. The device can be considered fully switched in this state. The angular separation between the $0^{\text {th }}$ and $1^{\text {st }}$ order is approximately $1.9^{\circ}$.

The device was characterized for its response to different polarization states of the incident light. Figure 4 shows the variation in power of the $0^{\text {th }}$ order for three different states of polarization of the incident light.

The curves labelled $0^{\circ}$ and $90^{\circ}$ correspond to the plane of polarization along and normal to the grating grooves, 
respectively. As expected, the response is quite independent of the polarization state of the incident light.



Fig. 4. Variation of power of the $0^{\text {th }}$ diffraction order of the grating for three different polarization states of light.

In order to realize polarization independent behaviour with conventional nematic liquid crystals usually a double stack has to be employed.

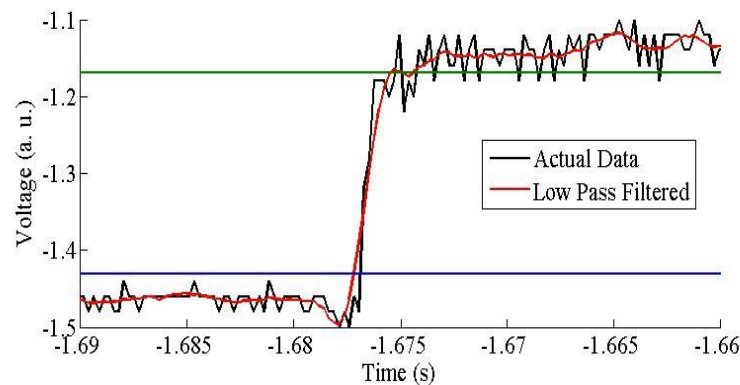

(a)

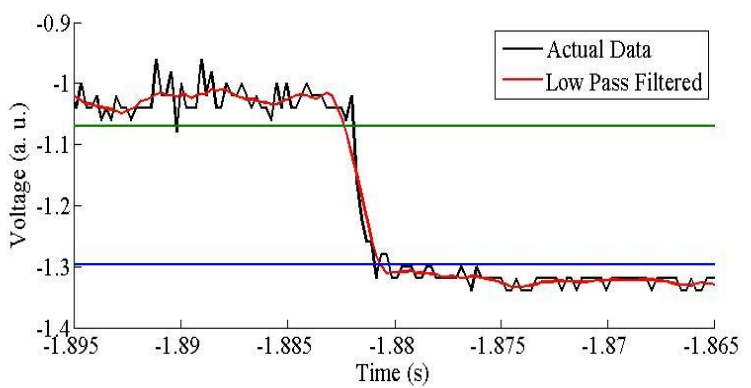

(b)

Fig. 5 Power in the $0^{\text {th }}$ diffraction order versus time for the PSBP-LC gratings (a) Switching on and (b) Switching off.

We also characterized the response times of these devices. The variation of the optical power recorded by the photodiode was fed to the oscilloscope and the time needed for the transmission to change from $10 \%$ to $90 \%$ was reduced. Figure 5 shows the switching behaviour of the device. The switching on and off times were $1.61 \mathrm{~ms}$ and $1.70 \mathrm{~ms}$, respectively. One remaining issue is the very high voltage requirement for such tunable devices. There are primarily three reasons behind such high voltage requirements: (i) The thickness of the cell is $\sim 20 \mu \mathrm{m}$. (ii) Because the dielectric constant of the grating material is four times smaller than that of the average dielectric constant value of the liquid crystals, there is a large voltage drop across the gratings. (iii) PSBP-LC inherently suffers from high switching voltage requirements. This shortcoming can be overcome by opting for polymer with higher dielectric constants and aiming for thinner devices. Yet another possibility is to use a transparent conductor deposited on top of the gratings or alternatively use a reflecting metal layer on top of gratings and use the device in reflective mode. Work in the direction of the aforementioned improvements is presently being carried out.

This work was partly supported by the Research FoundationFlanders (FWO) through the project No. 3GA04711, partly by the IWT through the SBO project SECONDOS, IWT- nr 120019, 2013-2016 and partly through the ICT COST Action IC1208 "Integrating devices and materials: a challenge for new instrumentation in ICT".

\section{References}

[1] D.C. Wright, et al., Rev. Mod. Phys. 61, 385 (1989).

[2] H. Kikuchi, et al., Nat. Mater. 1, 64 (2002).

[3] Samsung, Korea, SID exhibition, (2008).

[4] J. Yan, et al., Opt. Express 18, 11450 (2010).

[5] L. Rao, et al., Appl. Phys. Lett. 98, 081109 (2011).

[6] Y. Hisakado, et al., Adv. Mater. 17, 96 (2005).

[7] K. M. et al., J. Disp. Technol. 6, 49 (2010).

[8] Y. Chen, et al., Appl. Phys. Lett. 102, 251106 (2013).

[9] H. Choi, et al., Appl. Phys. Lett. 98, 131905 (2011).

[10] Y.H. Chen, et al., Opt. Express 19, 25441 (2011).

[11] Y. Li, et al., Opt. Express 19, 8045 (2011).

[12] C.T. Lee, et al., Opt. Express 19, 17402 (2011).

[13] Y.T. Lin, et al., J. Appl. Phys. 113, (2013).

[14] J.D. Lin, et al., Optics Express 22, 29479 (2014).

[15] W. Cao, et al., Nat. Mat. 1, 111 (2002).

[16] S.T. Hur, et al., Adv. Mater. 25, 3002 (2013).

[17] A. Mazzulla, et al., Soft. Mater. 8, 4882 (2012).

[18] C.W. Chen, et al., Opt. Express 20, 23978 (2012).

[19] O. Willekens, et al., Opt. Exp. 24, 8088 (2016).

[20] O. Willekens, et al., Opt. Exp. 24, 1541 (2016).

[21] M. Jazbinšek, et al., J. Appl. Phys. 90, 3831 (2001).

[22] C.C. Bowley, et al., Appl. Phys. Lett. 79, 9 (2001).

[23] Y.Q. Lu, et al., Appl. Phys. 95, 810 (2004).

[24] J.J. Butler et al., Opt. Lett. 25, 420 (2000).

[25] R.L. Sutherland et al., Appl. Phys. Lett. 64, 1074 (1994).

[26] X. Shang, et al., IEEE Photo. J. 7, 1 (2015).

[27] J. Yan, et al., Appl. Phys. Lett. 96, 071105 (2010).

[28] H.S. Chen, et al., Opt. Mat. Exp. 2, 1149 (2012).

[29] J. Yan. et al., Opt. Lett. 40, 4520 (2015).

[30] H.S. Chen, et al., Opt. Mat. Exp. 2, 1149 (2012).

[31] H.C. Cheng, et al., J. Disp. Technol. 8, 98 (2012). 